\title{
Biaxial strain-modified valence and conduction band offsets of zinc-blende GaN, GaP, GaAs, InN, InP, and InAs, and optical bowing of strained epitaxial InGaN alloys
}

\author{
Gus L. W. Hart \\ gus.hart@gmail.com \\ P. R. C. Kent
}

Alex Zunger

Follow this and additional works at: https://scholarsarchive.byu.edu/facpub

Part of the Astrophysics and Astronomy Commons, and the Physics Commons

\section{Original Publication Citation}

P. R. C. Kent, G. L. W. Hart, and A. Zunger, "Strain-modified energies of the valence and conduction band of zincblende $\mathrm{GaN}, \mathrm{GaP}, \mathrm{GaAs}, \operatorname{InN}, \mathrm{InP}$, InAs, and optical bowing of epitaxially strained and bulk-relaxed InGaN alloys," Appl. Phys. Lett. 814377 (2 Dec. 22). (Copyright (22) American Institute of Physics. This article may be downloaded for personal use only. Any other use requires prior permission of the American Institute of Physics.) The original article may be found here: http://apl.aip.org/resource/1/applab/v81/i23/p4377_s1

\section{BYU ScholarsArchive Citation}

Hart, Gus L. W.; Kent, P. R. C.; and Zunger, Alex, "Biaxial strain-modified valence and conduction band offsets of zinc-blende $\mathrm{GaN}, \mathrm{GaP}, \mathrm{GaAs}, \operatorname{lnN}$, InP, and InAs, and optical bowing of strained epitaxial InGaN alloys" (2002). Faculty Publications. 520.

https://scholarsarchive.byu.edu/facpub/520

This Peer-Reviewed Article is brought to you for free and open access by BYU ScholarsArchive. It has been accepted for inclusion in Faculty Publications by an authorized administrator of BYU ScholarsArchive. For more information, please contact ellen_amatangelo@byu.edu. 


\section{AIP Appiled Physics \\ Letters}

Biaxial strain-modified valence and conduction band offsets of zinc-blende GaN, GaP, GaAs, InN, InP, and InAs, and optical bowing of strained epitaxial InGaN alloys

P. R. C. Kent, Gus L. W. Hart, and Alex Zunger

Citation: Appl. Phys. Lett. 81, 4377 (2002); doi: 10.1063/1.1524299

View online: http://dx.doi.org/10.1063/1.1524299

View Table of Contents: http://apl.aip.org/resource/1/APPLAB/v81/i23

Published by the American Institute of Physics.

\section{Related Articles}

Competitive carrier interactions influencing the emission dynamics of GaAsSb-capped InAs quantum dots Appl. Phys. Lett. 101, 231109 (2012)

Observation of momentum space semi-localization in Si-doped $\beta-\mathrm{Ga2O} 3$

Appl. Phys. Lett. 101, 232105 (2012)

Role of the dimensionality of the [GaX]2 network in the Zintl phases EuGa2X2

J. Appl. Phys. 112, 103714 (2012)

An investigation of the Young's modulus of single-crystalline wurtzite indium nitride using an atomic force microscopy based micromechanical bending test

Appl. Phys. Lett. 101, 221906 (2012)

Temperature and Bi-concentration dependence of the bandgap and spin-orbit splitting in InGaBiAs/InP semiconductors for mid-infrared applications

Appl. Phys. Lett. 101, 221108 (2012)

\section{Additional information on Appl. Phys. Lett.}

Journal Homepage: http://apl.aip.org/

Journal Information: http://apl.aip.org/about/about_the_journal

Top downloads: http://apl.aip.org/features/most_downloaded

Information for Authors: http://apl.aip.org/authors

\section{ADVERTISEMENT}

\section{AIP Applied Physics Letters}

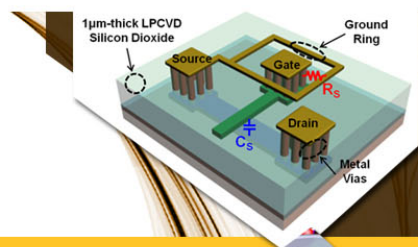

\section{SURFACES AND} INTERFACES

Focusing on physical, chemical, biological structural, optical, magnetic and electrical properties of surfaces and interfaces, and more...

\section{EXPLORE WHAT'S NEW IN APL}

SUBMIT YOUR PAPER NOW!
ENERCY CONVERSION AND STORACE 


\title{
Biaxial strain-modified valence and conduction band offsets of zinc-blende GaN, GaP, GaAs, InN, InP, and InAs, and optical bowing of strained epitaxial InGaN alloys
}

\author{
P. R. C. Kent, Gus L. W. Hart, and Alex Zunger ${ }^{\mathrm{a})}$ \\ National Renewable Energy Laboratory, Golden, Colorado 80401
}

(Received 3 July 2002; accepted 2 October 2002)

\begin{abstract}
Using density-functional calculations, we obtain the (001) biaxial strain dependence of the valence and conduction band energies of $\mathrm{GaN}, \mathrm{GaP}, \mathrm{GaAs}, \mathrm{InN}, \mathrm{InP}$, and InAs. The results are fit to a convenient-to-use polynomial and the fits provided in tabular form. Using the calculated biaxial deformation potentials in large supercell empirical pseudopotential calculations, we demonstrate that epitaxial strain reduces the InGaN alloy bowing coefficient compared to relaxed bulk alloys. (C) 2002 American Institute of Physics. [DOI: 10.1063/1.1524299]
\end{abstract}

The energy of the conduction band minimum (CBM) and valence band maximum (VBM) of common zinc-blende semiconductors can be altered ${ }^{1}$ via hydrostatic pressure, ${ }^{2}$ epitaxy-induced biaxial strain, ${ }^{3-6}$ or alloying. ${ }^{7}$ These energy changes are important parameters needed for the quantum design of electronic nanostructures. The rate of change of CBM and VBM energies with hydrostatic pressure ("absolute pressure deformation potentials") is summarized in Ref. 2 for many common binary semiconductors. However, reliable absolute biaxial deformation potentials are less common (e.g., Refs. 4 and 6). Here, we provide easy to use, fitted numerical results of the variation with (001) strain of the VBM and CBM energies of zinc-blende $\mathrm{GaN}, \mathrm{GaP}, \mathrm{GaAs}$, InN, InP, and InAs, obtained from first-principles localdensity calculations.

The VBM of zinc-blende materials consists of degenerate bands (two-fold $\Gamma_{8 v}$ and one-fold $\Gamma_{7 v}$ ) and, therefore, their response to strain is complex. ${ }^{8}$ Previously, first-order, linear in strain, perturbation models within the envelope function approximation have been used. ${ }^{3,7}$ Here, we calculate the band structure of each strained system self-consistently, so our results are not limited to small strains, envelope function approximations, or to low-order perturbative treatments of the strain-mediated interband coupling. Once obtained, the VBM and CBM energies are fit to a low-order polynomial in strain. Together with the $\operatorname{In} X / \mathrm{GaX}(X=\mathrm{N}, \mathrm{P}, \mathrm{As})$ unstrained valence band offset (tabulated in Ref. 9 for all common semiconductors), our results give the band offsets between $\mathrm{In} X / \mathrm{Ga} X$ at any intermediate (001) strain (i.e., corresponding to a substrate with in-plane lattice constant between that of $\operatorname{In} X$ and $\mathrm{Ga} X$ ).

The band structure and tetragonal deformations of strained zinc-blende materials were calculated in the localdensity approximation using the linear augmented plane wave (LAPW) approach ${ }^{10,11}$ (WIEN97 implementation), ${ }^{12}$ including spin-orbit effects. We use the exchange correlation of Perdew and Wang. ${ }^{13}$ For each in-plane (001) lattice constant $a_{\mathrm{InX}} \leqslant a_{\text {in-plane }} \leqslant a_{\mathrm{GaX}}$, we minimize the total energy with respect to the tetragonal distortion $c / a$. The band structure is then computed at $\left[a_{\text {in-plane }} ;(c / a)_{\text {eq }}\right]$ for a range of

${ }^{a)}$ Electronic mail: azunger@nrel.gov $a_{\text {in-plane }}$ values. To separate the movement of the VBM and CBM, we use the energy of the lowest $1 s$ states as a reference. (This is similar to both the experimental photoemission spectroscopy approach ${ }^{14,15}$ and first-principles calculational approach $^{16}$ to determining band offsets.) Although we use the local-density approximation for the conduction states, which are subject to the band gap error, we expect the change in the conduction state energies with lattice constant to be accurate.

The results for InP/GaP are plotted in Fig. 1 where we have aligned the unstrained eigenvalues using the calculated valence band offset ${ }^{9}$ and the unstrained experimental band gaps. ${ }^{17}$ Results are fit to polynomials of the form

$$
\begin{aligned}
& E_{\mathrm{SO}}=\Delta_{\mathrm{so}}+C_{1} x+C_{2} x^{2}+C_{3} x^{3}, \\
& E_{\mathrm{HH}}=C_{1} x+C_{2} x^{2}+C_{3} x^{3}, \\
& E_{\mathrm{LH}}=C_{1} x+C_{2} x^{2}+C_{3} x^{3}, \\
& E_{\mathrm{CBM}}=E_{\mathrm{gap}}+C_{1} x+C_{2} x^{2}+C_{3} x^{3},
\end{aligned}
$$

where $x \equiv\left(a_{\text {epi }}-a_{0}\right), a_{\text {epi }}$ is the in-plane lattice constant, $a_{0}$ is the cubic equilibrium lattice constant (in $\AA$ ), $\Delta_{\text {so }}$ is the spin-orbit splitting at the VBM interaction, and $E_{\text {gap }}$ is the band gap (in eV). This form for the band-edge states gives

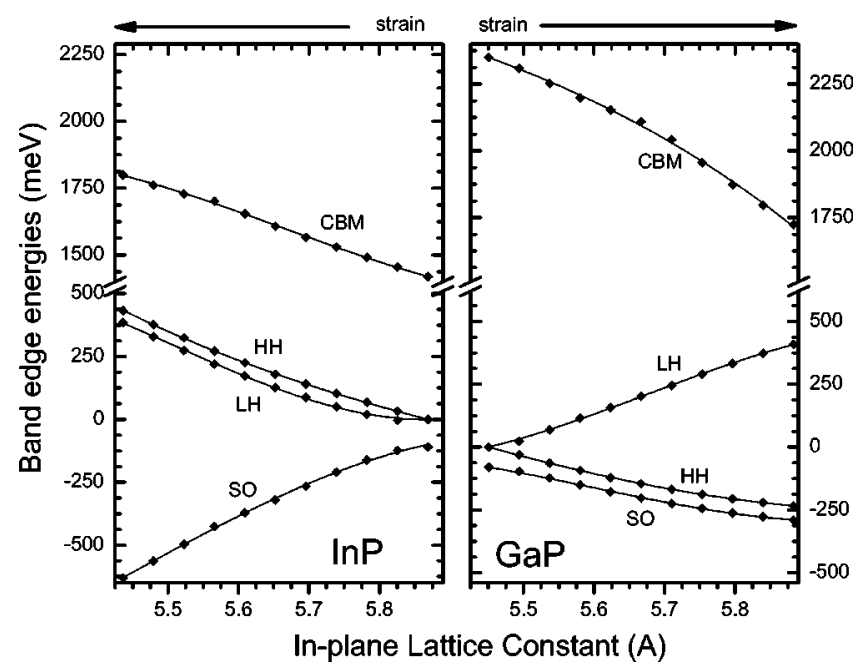

FIG. 1. LAPW calculated CBM and VBM states (indicated by points) for cubic GaP and InP for substrate lattice constants $a_{\mathrm{GaP}} \leqslant a_{\text {in-plane }} \leqslant a_{\mathrm{InP}}$. The corresponding fits from Table I are shown by solid lines. 
TABLE I. LDA fitted results according to Eqs. (1)-(4). The HH and LH states are labeled assuming the same valence band structure as InAs and GaAs.

\begin{tabular}{|c|c|c|c|c|}
\hline \multirow[b]{2}{*}{ Band-edge state } & & \multicolumn{3}{|c|}{ Parameters } \\
\hline & & $C_{1}$ & $C_{2}$ & $C_{3}$ \\
\hline \multirow[t]{4}{*}{$\mathrm{GaN}$} & SO & -558.0 & 1132.7 & -452.8 \\
\hline & LH & 1668.0 & -416.7 & 327.3 \\
\hline & $\mathrm{HH}$ & -593.9 & 1385.1 & -744.4 \\
\hline & CBM & -1071.6 & 1553.2 & -1122.8 \\
\hline \multirow[t]{4}{*}{$\mathrm{InN}$} & SO & 1584.7 & 228.5 & 1158.4 \\
\hline & LH & -602.3 & 286.3 & -1073.9 \\
\hline & $\mathrm{HH}$ & -674.7 & 157.6 & -1190.0 \\
\hline & CBM & -79.9 & 1748.3 & 1426.6 \\
\hline \multirow[t]{4}{*}{$\mathrm{GaP}$} & SO & -469.5 & -765.9 & 1699.0 \\
\hline & LH & 678.5 & 1686.7 & -2488.2 \\
\hline & $\mathrm{HH}$ & -777.8 & 433.3 & 274.7 \\
\hline & CBM & -972.2 & -788.9 & -814.1 \\
\hline \multirow[t]{4}{*}{$\mathrm{InP}$} & SO & 475.0 & -2748.5 & -2437.0 \\
\hline & LH & 6.8 & 3448.3 & 3155.4 \\
\hline & $\mathrm{HH}$ & -769.5 & 219.5 & -797.5 \\
\hline & CBM & -760.0 & 1020.0 & 1731.1 \\
\hline \multirow[t]{4}{*}{ GaAs } & SO & -245.1 & -959.9 & 1349.4 \\
\hline & $\mathrm{LH}$ & 531.1 & 531.6 & -439.6 \\
\hline & $\mathrm{HH}$ & -822.6 & 533.5 & -151.6 \\
\hline & CBM & -1828.7 & 469.6 & -244.9 \\
\hline \multirow[t]{4}{*}{ InAs } & SO & -53.9 & -3651.6 & -2364.5 \\
\hline & LH & 594.3 & 3412.6 & 2842.0 \\
\hline & $\mathrm{HH}$ & -873.7 & -778.3 & -1498.4 \\
\hline & CBM & -166.3 & 2515.7 & 4086.9 \\
\hline
\end{tabular}

the VBM as zero energy. The values for $a_{0}, \Delta_{\text {so }}$, and $E_{\text {gap }}$ can all be taken from the experiment; our fits provide the coefficients $C_{i}$. These coefficients are given in Table I, and the values of $a_{0}, \Delta_{\text {so }}$, and $E_{\text {gap }}$ are given in Table II.

In Fig. 2 we have plotted the difference (band offset) in light hole (LH), heavy hole (HH), and split-off (SO) energies betweeen $\mathrm{GaP}$ and $\mathrm{InP}$ versus lattice constant. From this, we can see that while the unstrained $\mathrm{LH}$ and $\mathrm{HH}$ offsets between $\mathrm{GaP}$ and $\mathrm{InP}$ are $110 \mathrm{meV}$, these change to $41 \mathrm{meV}$ and 438 $\mathrm{meV}$ (for the $\mathrm{LH}$ and $\mathrm{HH}$, respectively) when $\mathrm{GaP}$ and $\mathrm{InP}$ are grown on a GaAs substrate (indicated by the vertical dotted line in Fig. 2). Similarly, the offset for the SO states changes from -20 to $-190 \mathrm{meV}$. We show the LAPW calculated CBM, VBM, and fitted results for InAs/GaAs in Fig. 3, and for InN/GaN in Fig. 4.

The strain-modified band-edge energies are important for understanding the difference in alloy optical bowing of

TABLE II. Experimental lattice constants and band structure parameters (from Ref. 17).

\begin{tabular}{lccc}
\hline \hline & \multicolumn{3}{c}{ Parameters } \\
\cline { 2 - 4 } Material & $a_{0}(\mathrm{~A})$ & $\Delta_{\text {So }}(\mathrm{meV})$ & $E_{\text {gap }}(\mathrm{eV})$ \\
\hline $\mathrm{GaN}$ & 4.508 & 11 & 3.5 \\
$\mathrm{InN}$ & 4.979 & 11 & 1.9 \\
$\mathrm{GaP}$ & 5.451 & 80 & 2.35 \\
$\mathrm{InP}$ & 5.869 & 110 & 1.42 \\
$\mathrm{GaAs}$ & 5.652 & 340 & 1.519 \\
$\mathrm{InAs}$ & 6.058 & 380 & 0.418 \\
\hline \hline
\end{tabular}

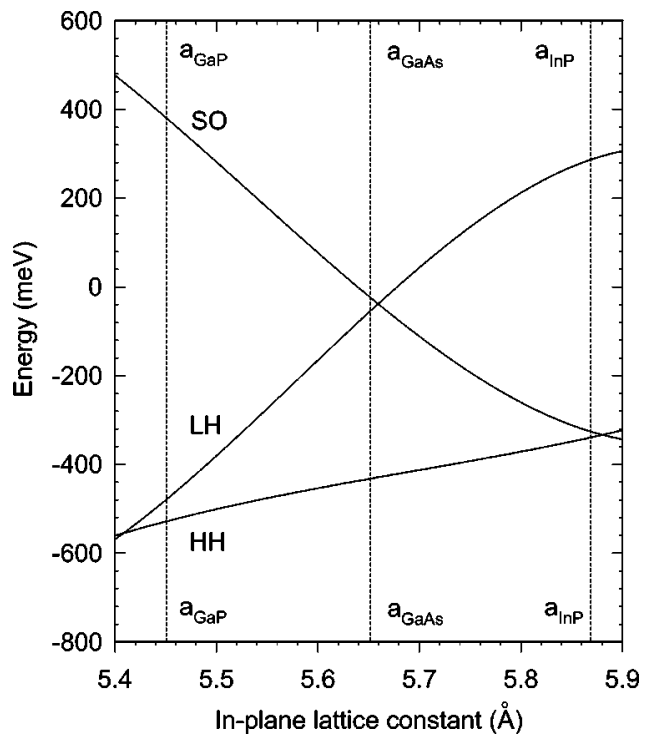

FIG. 2. Valence band offsets between $\mathrm{GaP}$ and $\mathrm{InP}$ for substrate lattice constants $a_{\mathrm{GaP}} \leqslant a_{\text {in-plane }} \leqslant a_{\mathrm{InP}}$, for LH, HH, and SO states.

freestanding, relaxed "bulk" systems and epitaxially strained films. To illustrate this, we examined the case of cubic $\mathrm{In}_{x} \mathrm{Ga}_{1-x} \mathrm{~N}$ under bulk and epitaxial (on $\mathrm{GaN}$ ) conditions. We first calculated the composition variation of the VBM and CBM energies of the bulk alloys. We generated straindependent empirical pseudopotentials following the procedure of Ref. 18. Specifically, the potentials were fitted to the local density approximation calculated absolute pressure and biaxial deformation potential (described herein), as well as to the measured band structure. The bulk alloy was modeled by a translationally repeated 13824 atom cubic supercell whose 6912 cation sites are randomly occupied by In and Ga atoms, while all the nitrogen atoms are placed at their proper tetrahedral site. Atomic positions were relaxed by minimizing the strain energy while keeping the overall lattice constant at its Vegard value. The band structure was calculated using a plane-wave basis, and the resulting band-edge energies averaged over five randomly selected supercell configurations. The solid lines in Fig. 5(a) show the band-edge energies, and

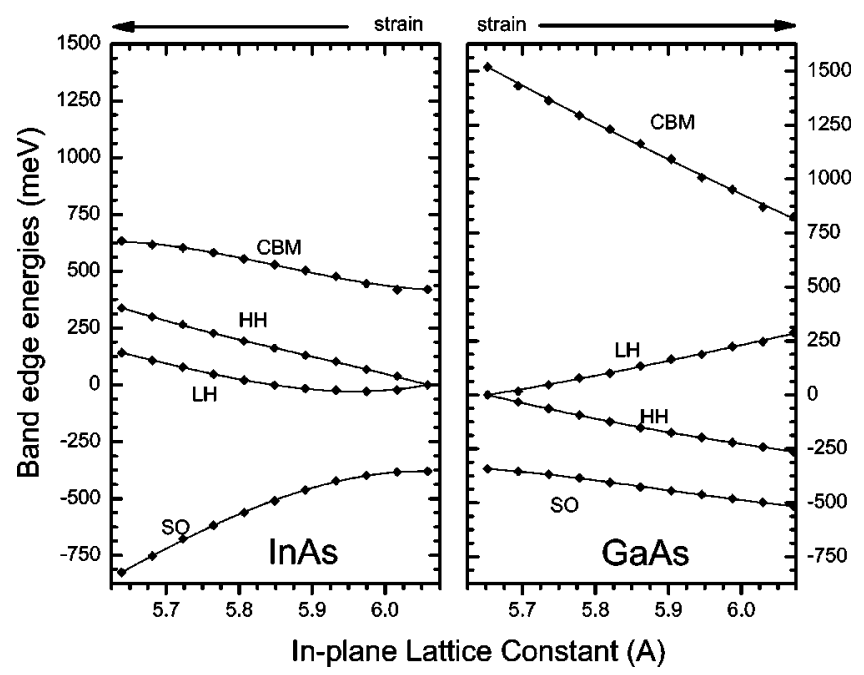

FIG. 3. LAPW calculated CBM and VBM states (indicated by points) for cubic GaAs and InAs for substrate lattice constants $a_{\mathrm{GaAs}} \leqslant a_{\text {in-plane }} \leqslant a_{\mathrm{InAs}}$. The corresponding fits from Table I are shown by solid lines. 


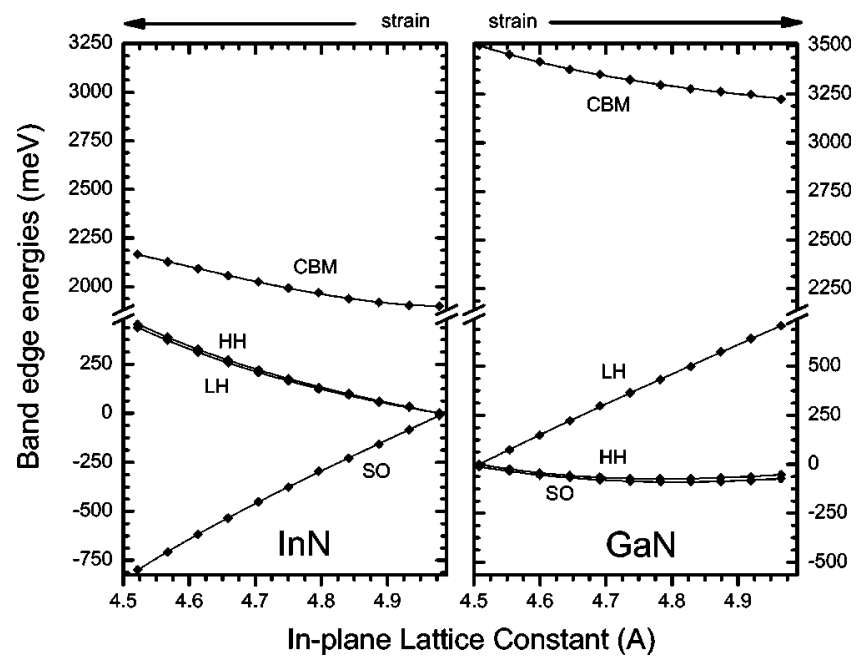

FIG. 4. LAPW calculated CBM and VBM states (indicated by points) for cubic GaN and InN for substrate lattice constants $a_{\mathrm{GaN}} \leqslant a_{\text {in-plane }} \leqslant a_{\mathrm{InN}}$. The corresponding fits from Table I are shown by solid lines. The unstrained valence band offset between $\mathrm{InN}$ and $\mathrm{GaN}$ is $260 \mathrm{meV}$.

are quantitatively similar to Bellaiche et al. ${ }^{19}$ except for improved statistics in the present work. The strong upward shift of the VBM for indium concentrations $<10 \%$ is consistent with recent X-ray measurements. ${ }^{20}$ In Fig. 5(b), we show the band gap bowing coefficient assuming a gap of $1.9 \mathrm{eV}$ for InN. We see that the bowing coefficient is composition dependent and is large and positive, $\geqslant 4 \mathrm{eV}$, for indium compositions below $10 \%$, due to the strong upward bowing of the VBM. For higher indium compositions, the bowing is reduced to $2-3 \mathrm{eV}$. When we assume in our pseudopotential fit that $\mathrm{InN}$ has a gap of $0.8 \mathrm{eV}$, the bowing coefficient is reduced significantly for both bulk and epitaxial film but the relative bowing is unchanged.

To model the epitaxial alloy grown on $\mathrm{GaN}$, we confined the in-plane lattice constant of the $\operatorname{In}_{x} \mathrm{Ga}_{1-x} \mathrm{~N}$ alloy to that of $\mathrm{GaN}$, relaxing the $c / a$ ratio and all atomic positions. The band-edge energies, obtained using the same method as for the bulk alloys, are shown in Fig. 5(a) as dashed lines. Now the bowing coefficients are reduced, compared to the bulk, for indium compositions up to $\sim 30 \%$, e.g., $b=2.8$ versus $3.4 \mathrm{eV}$ for the epitaxial and bulk bowing coefficient at $x$ $=0.20$. In Ref. 21 , a reduction in bowing coefficient due to expitaxy of $0.7 \mathrm{eV}$ was measured for $x<0.25$.

To understand the effect of epitaxy-reduced alloy bowing, we refer again to Fig. 4. We see that compressing $\mathrm{InN}$ biaxially to the in-plane lattice constant of $\mathrm{GaN}$ raises the VBM energy by $480 \mathrm{meV}$ and also raises the CBM by 350 $\mathrm{meV}$, so the epitaxial gap is only $130 \mathrm{meV}$ lower than the bulk InN gap. At the other end of the composition range, $x$ $=0$, the bulk and epitaxial gaps are identical. As the In composition increases from $x=0$, the epitaxial alloy reduces its band gap by a lesser amount than the bulk alloy since the GaN component has a higher VBM, while the InN component has a lower VBM with increasing $a_{\text {in-plane }}$. On the other hand, the CBM of both the GaN and InN components decrease as $a_{\text {in-plane }}$ increases. However, the CBM of the bulk alloy decreases faster than that of the epitaxial alloys. Consequently, the epitaxial alloy has a smaller optical bowing than the bulk alloy. This observation shows that larger bow-
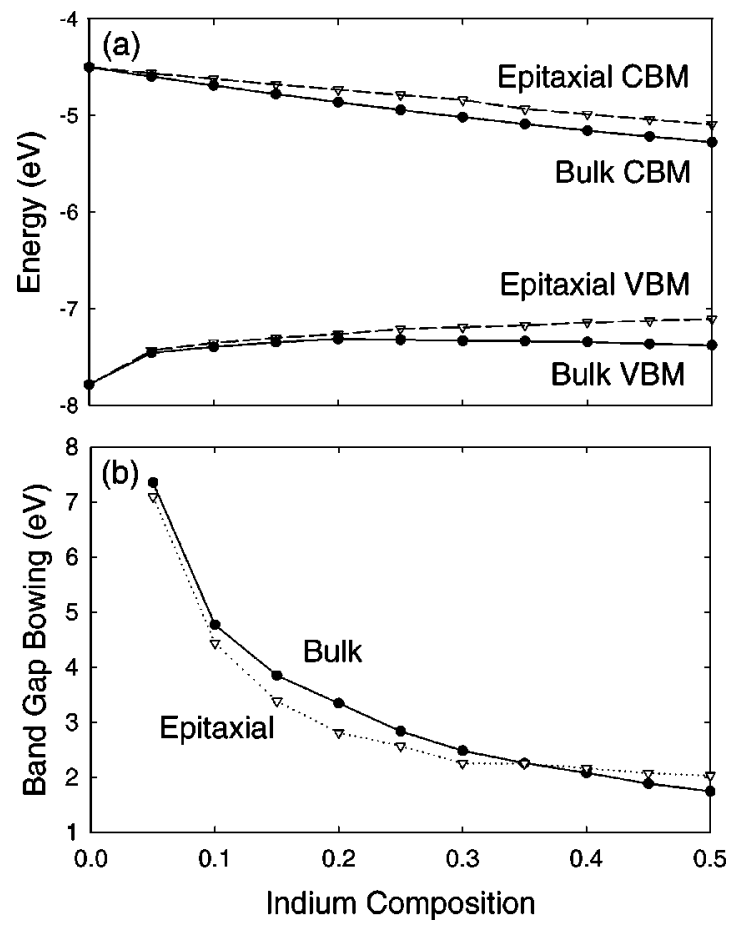

FIG. 5. Band edges and band gap bowing of bulk and epitaxially strained InGaN calculated using the empirical pseudopotential method, assuming a gap of $1.9 \mathrm{eV}$ for $\mathrm{InN}$.

ing is expected in relaxed, bulklike samples, while epitaxial samples should have $\sim 0.5 \mathrm{eV}$ lower bowing parameters, for the device-relevant indium composition range of $\leqslant 30 \%$. This holds for InN gap of either 1.9 or $0.8 \mathrm{eV}$.

This work is supported by the U.S. Department of Energy, SC-BES-OER Grant No. DE-AC36-98-GO10337.

${ }^{1}$ P. Y. Yu and M. Cardona, Fundamentals of Semiconductors (Springer, New York, 1996).

${ }^{2}$ S.-H. Wei and A. Zunger, Phys. Rev. B 60, 5404 (1999).

${ }^{3}$ F. H. Pollak and M. Cardona, Phys. Rev. 172, 816 (1968).

${ }^{4}$ C. G. Van de Walle and M. Martin, Phys. Rev. B 34, 5621 (1986).

${ }^{5}$ S.-H. Wei and A. Zunger, Phys. Rev. B 57, 8983 (1998).

${ }^{6}$ C. G. Van de Walle, Phys. Rev. B 39, 1871 (1989).

${ }^{7}$ G. L. Bir and G. E. Pikus, Symmetry and Strain-Induced Effects in Semiconductors (Wiley, New York, 1974).

${ }^{8}$ Y. Zhang, Phys. Rev. B 49, 14352 (1994).

${ }^{9}$ S.-H. Wei and A. Zunger, Appl. Phys. Lett. 72, 2011 (1998).

${ }^{10}$ S.-H. Wei and H. Krakauer, Phys. Rev. Lett. 55, 1200 (1985), and references therein; D. J. Singh, Planewaves, Pseudopotential, and the LAPW Method (Kluwer, Boston, 1994), and references therein.

${ }^{11}$ D. J. Singh, Phys. Rev. B 43, 6388 (1991).

${ }^{12}$ P. Blaha, K. Schwarz, and J. Luitz, WIEN97, Vienna University of Technology, Vienna, 1997; Updated version of P. Blaha, K. Schwarz, P. Sorantin, and S. B. Trickey, Comput. Phys. Commun. 59, 399 (1990).

${ }^{13}$ J. P. Perdew and Y. Wang, Phys. Rev. B 45, 13244 (1992).

${ }^{14}$ S. P. Kowalczyk, J. T. Cheung, E. A. Kraut, and R. W. Grant, Phys. Rev. Lett. 56, 1605 (1986); C. Hsu and J. P. Faurie, ibid. 58, 1127 (1987).

${ }^{15}$ T. M. Duc, C. Hsu, and J. P. Faurie, Phys. Rev. Lett. 58, 1127 (1987).

${ }^{16}$ G. L. W. Hart and A. Zunger, Phys. Rev. B 62, 13522 (2000).

${ }^{17}$ Landolt-Börnstein, Numerical Data and Functional Relationships in Science and Technology, (Springer, Berlin, 1987), Vol. 22a.

${ }^{18}$ K. Kim, P. R. C. Kent, A. Zunger, and C. B. Geller, Phys. Rev. B 66, 045208 (2002).

${ }^{19}$ L. Bellaiche, T. Mattila, L.-W. Wang, S.-H. Wei, and A. Zunger, Appl. Phys. Lett. 74, 1842 (1999).

${ }^{20}$ P. Ryan, C. McGuinness, J. E. Downes, and K. E. Smith, Phys. Rev. B 65, 205201 (2002).

${ }^{21}$ C. A. Parker, J. C. Roberts, S. M. Bedair, M. J. Reed, S. X. Liu, N. A. El-Masry, and L. H. Robins, Appl. Phys. Lett. 75, 2566 (1999). 